\title{
Evaluation of the Serrated Lesions Detection Rate and Its Role as a Colonoscopy Quality Criteria
}

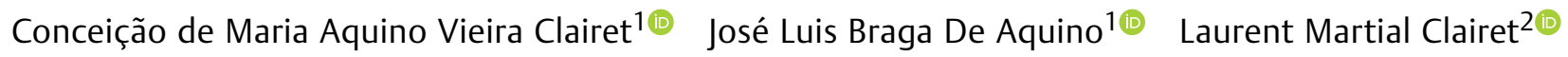 \\ 1 Pontifícia Universidade Católica de Campinas, Campinas, SP, Brazil \\ ${ }^{2}$ Centro Universitário UNIFACID, Teresina, PI, Brazil \\ Address for correspondence Conceição de Maria Aquino Vieira \\ Clairet, MD, Rua Desembargador Manoel Castelo Branco, 1400, Ed Via \\ J Coloproctol 2021;41(3):228-233. \\ Doccia apt 1303, Bairro Jockey, CEP: 64049270, Teresina, PI, Brazil \\ (e-mail: vieira_conceicao@yahoo.com.br).
}

\begin{abstract}
Keywords

- colonoscopy

- colorectal cancer

- polyps

Objectives To evaluate the serrated lesion detection rate in colonoscopy at a specialized clinic and its role as quality criteria for endoscopic examination.

Methods This is an observational cross-sectional study with all patients that underwent colonoscopy between October 2018 and May 2019, performed by an experimented physician. A questionnaire was answered before the examination by the patient, and another questionnaire after the colonoscopy was answered by the medical team. All polyps identified were removed and sent to the same pathologist for analysis. Results A total of 1,000 colonoscopies were evaluated. The average age of the patients was 58.9 years old, and most of them were female (60.6\%). In $62.5 \%$ of the procedures, polyps were removed, obtaining a total of 1,730 polyps, of which 529 were serrated lesions, being 272 sessile serrated lesions (SSL). This data resulted in a serrated lesion detection rate (SDR) of $29.2 \%$, and of $14 \%$ when considering only the SSL detection rate (SSLDR). The right colon had higher rates, with $22.3 \%$ SDR and $15.3 \%$ SSLDR. Screening colonoscopies also presented a higher serrated detection rate, of $20 \%$, followed by diagnostics and follow-up exams. Smoking was the only risk factor associated with higher serrated detection rate.

Conclusions The serrated lesion detection rate is higher than the ones already previously suggested and the have the higher rates were stablished in the right colon and on screening exams.
\end{abstract}

\section{Introduction}

Colorectal cancer (CRC) is the third most common cause of cancer, when excluding skin cancer, and the fourth most common cause of death by cancer worldwide. There were $\sim$ 1.8 million new cases in 2018, and $>800,000$ deaths in the same year, corresponding to $10.2 \%$ of all cancers diagnosed. ${ }^{1}$

From a morphologic point of view, CRC develops following two main pathways: adenomatous and serrated. The first one is well known and studied by the medical community; it arises through an adenoma-carcinoma sequence and is responsible for $60 \%$ of sporadic cancers. ${ }^{2}$

The second one, the serrated pathway, is gaining notoriety recently and is considered responsible for between 20 and $30 \%$ of sporadic CRCs. It has, in the epigenetic instability, also known as CpG Island Methylator phenotype, its main mechanism. ${ }^{3,4}$ Serrated lesions (SL) are the precursors of this pathway and are classified as: hyperplastic polyps (HP), which usually are $<5 \mathrm{~mm}$, flat, and have a star-shaped crypt received

September 16, 2020

accepted

January 18, 2021
DOI https://doi.org/

10.1055/s-0041-1730261.

ISSN 2237-9363.

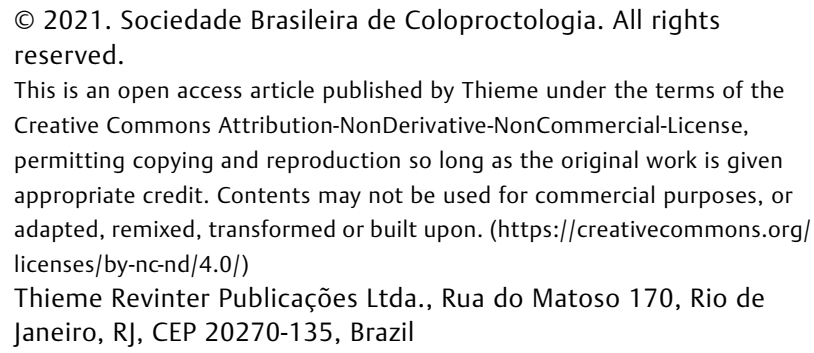

This is an open access article published by Thieme under the terms of the Creative Commons Attribution-NonDerivative-NonCommercial-License, permitting copying and reproduction so long as the original work is given appropriate credit. Contents may not be used for commercial purposes, or adapted, remixed, transformed or built upon. (https://creativecommons.org/ licenses/by-nc-nd/4.0/)

Thieme Revinter Publicações Ltda., Rua do Matoso 170, Rio de Janeiro, RJ, CEP 20270-135, Brazil 
when identified with chromoendoscopy; sessile serrated lesions (SSL) with or without dysplasia, which are also flat, covered with a mucus cap, may present depressed areas, have an open shaped crypt, cloud shape surfaces, and irregular boundaries. Traditional serrated adenoma (TSA) and unclassified serrated adenoma are usually pedunculated and similar to conventional adenoma lesions., 5

Colonoscopy has high sensibility and specificity and is considered the gold standard test for CRC screening in the United States. Patients that underwent at least one colonoscopy in the past 10 years have a much lower risk of having CRC. ${ }^{7}$

Besides the progress achieved with colonoscopy in preventing CRC, between 5 and $9 \%$ of the patients with this malignant neoplasm have had a colonoscopy considered as normal performed in the past 3 years that preceded their diagnosis. Therefore, they were labeled as carriers of interval cancer. $^{8}$

Interval cancer is strongly related to SL, since the serrated pathway leads to CRC in a shorter period and these types of lesions are usually flat, depressed, and covered with mucus cap, which makes them harder to be identified and removed. ${ }^{9}$ The location in the proximal colon and the presence of epigenetic instability are also factors that reassure the association between SL and this type of cancer. ${ }^{10}$

The literature shows that the detection of SL varies significantly and is endoscopist-related. ${ }^{11}$ It has been noticed that the prevalence of these lesions, especially of SSLs, which have a higher malignant potential, presents an important variation among medical centers and endoscopists, so the numbers presented in the literature seem to be lower than those observed on a daily practical basis. ${ }^{12}$

It is also known that the risk of advanced adenomas is lower in patients who have undergone a previous colonoscopy, although this same effect is not seen in patients with SL; therefore, colonoscopy shows to be more effective in the prevention of cancers arising from the adenomatous pathway than from the serrated pathway. ${ }^{13}$

The establishment of an endoscopic quality criteria that quantifies the adenoma detection rate (ADR) turned up to be really effective in obtaining good results regarding the prevention of CRC emerging from the adenomatous pathway. ${ }^{7,8}$

However, for the SL, a detection rate to be achieved has not been defined yet. In this scenario, some studies indicate that more than half of the SLs in the right colon are not identified by most endoscopists, and that, in average, $25 \%$ of the lesions are not detected. ${ }^{14}$

This important gap between data from different medical centers and specialists shows the need to bring awareness to the importance of proper diagnosing and removal of SLs. The establishment of a minimum rate to be reached could encourage doctors and help to improve these data. ${ }^{15}$

In the present research, we aimed to evaluate the serrated lesions detection rate (SDR) in colonoscopies performed in a specialized center and its role as a quality criterion in endoscopic exams.

\section{Methods}

A cross-sectional observational study was performed with patients that underwent colonoscopy in a specialized center in Campinas, state of São Paulo, Brazil, between October 2018 and May 2019, by one experimented endoscopist.

The present study was approved by the ethical committee of research in human beings from PUC-CAMPINAS, under the file number 2.908.718, and all patients were asked to sign a Free and Informed Consent Term.

The exams were executed following the American Society for Gastrointestinal Endoscopy (ASGE) ${ }^{16}$ quality criteria and had imaging enhanced technology used or second forward view examination performed, when necessary.

Data was compiled with help from two questionnaires, one answered by the patients, informing about symptoms, alcohol intake, previous colonoscopies, family history of CRC, obesity, smoking habits, diabetes, physical activity, and other variables.

A second questionnaire was answered by the medical team at the end of the exams, providing information about the lesions removed, the Boston Bowel Preparation Scale, the duration of the procedure, the withdraw time, among other variables.

All lesions identified were sent to be analyzed by a single gastrointestinal expert pathologist, except from the nonremovable ones, for which biopsies were performed. They were classified by proximal or distal location to the splenic flexure. Hyperplastic areas on the sigmoid and rectum, defined as areas with multiple diminutive polyps with hyperplastic aspect shown by enhanced imaging techniques, were not removed or biopsied, following the ASGE recomandations. ${ }^{17}$

Patients $<18$ years old, those who did not accept to be a part of the research, who did not sign the consent term, who did not answer the questionnaire or did so in an incomplete way were excluded from the research. Colonoscopies with a score $<6$ in the Boston Scale, incomplete, not reaching the cecum, and with $<6$ minutes of withdraw time were also removed from data.

For all the statistics analysis, the significance level was defined as $5 \%$. The Student $t$-test was used to compare averages; the chi-squared test and the Poisson regression were used for univariate data analysis. IBM SPSS Statistics for Windows (IBM Corp., Armonk, NY, USA), R software (R Foundation, Vienna, Austria), and Microsoft Excel (Microsoft Corp., Redmond, WA, USA) were chosen to assist on the analysis.

\section{Results}

During the predetermined period, 1,000 colonoscopies fulfilled the inclusion criteria for the research.

The mean age of the patients was 58.9 years old, with a standard deviation (SD) of 13,2 years; $60,6 \%$ of the patients were female.

Polypectomies were performed in $62,5 \%$ of the patients, providing a total of 1,730 polyps. Adenomatous polyps (AP) 
Table 1 Polyps detection rate by histological type and patient gender

\begin{tabular}{|l|l|l|l|l|}
\hline & & Gender & & \\
\hline Detection Rate & Combined & Female & Male & p-value \\
\hline ADR & $51.7 \%$ & $48.5 \%$ & $56.5 \%$ & 0.961 \\
\hline SDR & $29.2 \%$ & $28.8 \%$ & $30.4 \%$ & \\
\hline SSLDR & $14.0 \%$ & $15.6 \%$ & $11.4 \%$ & \\
\hline
\end{tabular}

Abbreviations: ADR, adenoma detection rate; SDR, serrated lesion detection rate; SSLDR, sessile serrated lesion detection rate.

represented $69,5 \%$ of all polyps. A total of 529 lesions were SLs, including HPs and SSLs, which corresponded to $30.5 \%$ of the polyps. No TSA was diagnosed. Sessile serrated lesions had a prevalence of $15.7 \%$. Adenocarcinoma was found in $0.9 \%$ of the patients.

The combined ADR was of $51.7 \%$, being $56.5 \%$ among males and $48.5 \%$ among females.

At least 1 SL was identified in 292 patients, providing an SDR of $29.2 \%$, as shown in - Table 1. Considering only SSLs, the combined detection rate was of $14.0 \%$, with $15.6 \%$ in females and $11.4 \%$ in males; however, there was no statistical significance that related gender and a higher SSLDR $(p=0.961)$.

The diagnosis investigation was the indication for colonoscopy in $48 \%$ of the patients, surveillance was responsible for $40 \%$ of the exams, and CRC screening for only $12 \%$ of the exams. According to - Table 2, the SSLDR is highest on screening exams $(20,0 \%)(p=0.015)$.

Regarding the location on the colon, APs were found in the proximal colon in $69.1 \%$ of the cases. In the SL group, $55.1 \%$ were in the proximal colon, and when only SSLs were considered, $84,9 \%$ were in the proximal colon. As demonstrated, there was a higher possibility of identifying an SSL in the proximal colon, as seen on - Fig. $1(p<0.001)$.

The detection rates calculated are shown in - Table 3. We can observe that, when calculated for SLs, the SDR was of $22.3 \%$ and the SSLDR was of $15.3 \%$ on the proximal colon, emphasizing that location in the proximal colon is related to a higher SSLDR $(p<0.001)$.

Patients with multiple diminutive polyps in the rectum that appeared to be hyperplastic, as in - Fig. 2, did not have biopsies performed; therefore, they were not considered as having SLs. This scenario was found in $10 \%$ of the patients.
Through a univariate analysis of risk factors linked with SLs, only smoking was associated with a higher risk of developing SLs (PR: 1.18; 95\% confidence interval [CI]: 1.00-1.40). Skin color, obesity, alcohol intake, physical activity, diabetes, and use of anti-inflammatories did not present statistical significance.

\section{Discussion}

Serrated lesions were seen as benign until a few years ago. They represent a challenge for the endoscopists nowadays, corresponding to up to $30 \%$ of sporadic CRCs. ${ }^{7}$ The flatter shape, the fact that they are more often located in the proximal colon, and the presence of a mucus cap covering theses lesions make their identification during colonoscopy quite difficult. $^{18}$

With a well-defined role on the prevention of CRC, colonoscopy stills needs improvement so that we can identify even more lesions and downsize the impact of interval cancer.

The SDR, besides being as important as the ADR in the prevention of CRC, still does not have a value to be reached defined by specialists, and the attempts of suggesting values varies from 1.5 to $10 \%$ in the literature. ${ }^{19,20}$ According to the data obtained in our study, we can imply that performing colonoscopy following quality criteria, with high-definition colonoscopes, and by an experimented endoscopist, the lesion detection rate will be much higher than expected.

In our data, the ADR was of $51.7 \%, 2$ times higher than the minimum established by the ASGE. ${ }^{16}$ When evaluating SLs, the SDR was of $29.2 \%$, and in 140 patients, at least 1 SSL, which has a higher malignifying potential, was detected, establishing an SSLDR of $14.0 \%$.

According to previous studies, the prevalence of SLs varies considerably. ${ }^{12,21,22}$ In a review from researches performed between 2003 and 2014, the prevalence varied from 0.6 to $5.3 \% .{ }^{23}$ In a systematic review, the average prevalence was of $4 \%$ and it varied from 0 to $20 \%{ }^{24}$

There were some attempts to develop an SDR, as it was done for the adenomas. A multicenter study suggested a $5 \%$ SDR, considering only proximal colon lesions; the endoscopists with best results had an SDR of up to $20 \%,{ }^{20}$ while in our study we had a $22.3 \%$ SDR considering the same findings. In contrast, Vleugels et al. came up with a $10 \%$ value of SDR to be pursued; ${ }^{25}$ however, considering lesions from the whole colon, our results demonstrated a 29.2\% SDR in this scenario.

Table 2 Polyp detection rate by polyp histological type and exam indication

\begin{tabular}{|c|c|c|c|c|c|c|c|}
\hline \multirow[b]{3}{*}{ Detection Rate } & \multicolumn{6}{|c|}{ Indication } & \\
\hline & \multicolumn{2}{|c|}{ Screening } & \multicolumn{2}{|c|}{ Surveillance } & \multicolumn{2}{|c|}{ Diagnosis } & \multirow[b]{2}{*}{ p-value } \\
\hline & $n$ & $\%$ & $n$ & $\%$ & $n$ & $\%$ & \\
\hline ADR & 77 & $64.16 \%$ & 261 & $65.25 \%$ & 199 & $41.45 \%$ & 0.818 \\
\hline $\begin{array}{l}\text { SDR } \\
\end{array}$ & 43 & $35.83 \%$ & 139 & $34.75 \%$ & 111 & $23.12 \%$ & 0.621 \\
\hline SSLDR & 24 & $20.0 \%$ & 60 & $15.0 \%$ & 55 & $11.45 \%$ & 0.015 \\
\hline
\end{tabular}

Abbreviations: ADR, adenoma detection rate; SDR, serrated lesion detection rate; SSLDR, sessile serrated lesion detection rate. 

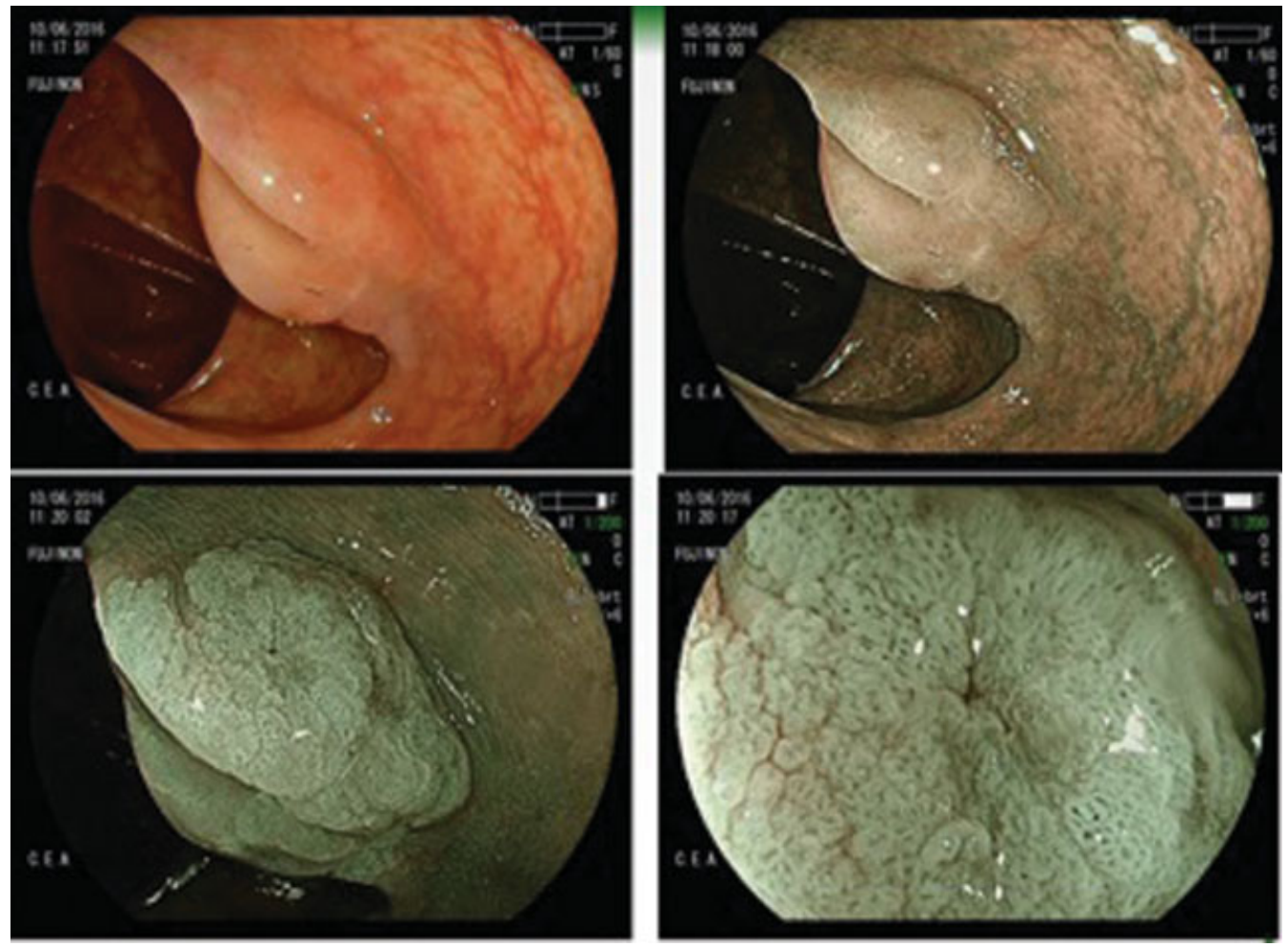

Fig. 1 Sessile serrated lesion identified on the proximal colon; colonoscopy performed with assistance of Blue Light Imaging technology.

Table 3 Polyp detection rate by the location in the colon

\begin{tabular}{|c|c|c|c|}
\hline & \multicolumn{2}{|l|}{ Location } & \multirow[b]{2}{*}{ p-value } \\
\hline & Proximal colon & Distal colon & \\
\hline Detection Rate & $\%$ & $\%$ & $<0.001$ \\
\hline ADR & $42.3 \%$ & $24.8 \%$ & \\
\hline SDR & $22.3 \%$ & $10.8 \%$ & \\
\hline SSLDR & $15.3 \%$ & $2.5 \%$ & \\
\hline
\end{tabular}

Abbreviations: ADR, adenoma detection rate; SDR, serrated lesion detection rate; SSLDR, sessile serrated lesion detection rate.

Another research suggested an 11\% SDR and a 7\% SSLDR, and concluded that using the SDR with all the SLs was easier to adjust to a daily basis, without damaging the results, given that both rates are directly related. ${ }^{19}$ Besides suggesting values much lower than those we have identified, this conclusion does not transfer to our reality, considering that we had a very important difference between a $29.2 \%$ SDR and a $14 \%$ SSLDR, even though they are correlated $(p<0.001)$. Using only the SDR to evaluate the endoscopist's performance, considering all SLs, could generate a false sense of prevention, since these lesions have different malignant potential, and a high SDR could be secondary to a high prevalence of HPs that are not the main focus on preventing CRC arising from the serrated pathway.

Analyzing several studies available, it is clear that, before setting an SDR, it is mandatory to define which sample of lesions and patients will be considered. The different methodologies used, considering different types of SLs and the

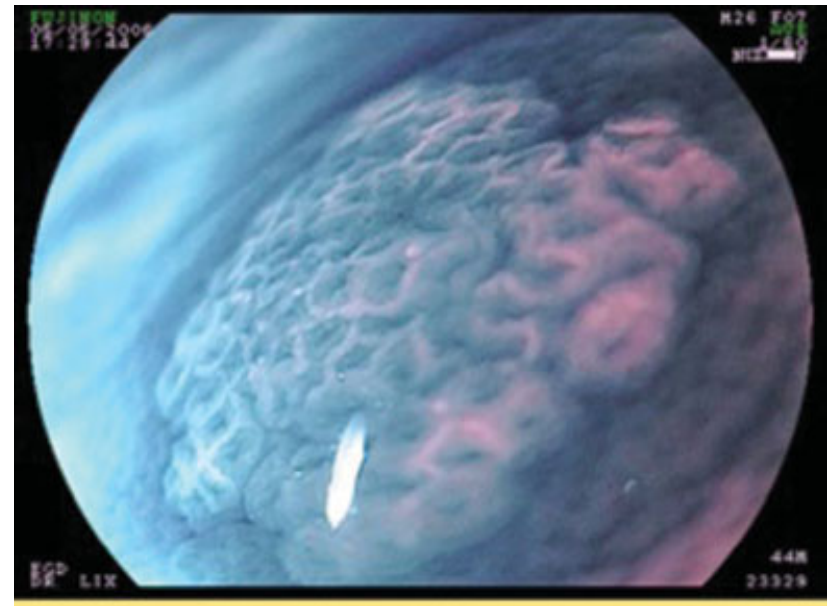

Fig. 2 Hyperplastic polyp diagnosed with chromoendoscopy and enhanced imaging technique.

location on proximal or on the whole colon, all these points makes the comparisons between data hard to be unbiased.

Another point that needs to be highlighted is that the population studied to define ADR consisted of screening patients; in other words, asymptomatic patients, aged $>50$ years old, and who were undergoing their first colonoscopy. This is a limited population, which makes it harder for the endoscopist to use the same data on their practice.

Anderson et al. compared a group of screening colonoscopies with surveillance ones. While there was an important difference in the detection rate for adenomas, the same difference was not significant when the SDR was evaluated. ${ }^{13}$ 
Therefore, using an SDR in a common population would be an option.

However, in our study, when the SSLDR was evaluated only on screening patients, a higher value was found (20.0\%) $(p=0.015)$. In this scenario, using a screening population to define the detection rate would generate a higher rate to be achieved than the one expected to be found in a common population.

When considering results from studies that evaluated the performance of a single experimented endoscopist, in 2 of them the ADRs were of 47.8 and $48 \%$, and the SSLDRs were of 8.1 and $20 \% .^{23,26}$ Results from a single doctor experiment, just like the present study, seems to present a higher SSLDR. Gathering information from only one endoscopist provides more control of the variants that could generate bias, such as the colonoscopy techniques applied, the quality of the exam performed, as well as the level of experience of the examiner; as Schramm et al. described, the personal ability of the endoscopist has an important role in having a high SSLDR. ${ }^{27}$

The literature shows that endoscopists with higher ADRs tend to have higher SDRs and SLLDRs, which could be one of the reasons for finding higher SDRs and SSLDRs in our data. Considering that the ADR was two times higher than the benchmark, a bigger attention to seek adenomas would also allow to identify more SLs. ${ }^{21,28}$ The use of image-enhancing technologies, when there was a doubt regarding the features of the mucosa, is also a factor that could have increased the detection rates, especially of flat lesions that could have been undetected. $^{9}$

Female patients had a higher SDR then males, 15.6 and $11.4 \%$, respectively, but this difference did not have statistical significance $(p=0.961)$. Prior studies have divergent results regarding a higher prevalence of SLs among males or females. Tumors with epigenetic instability, a factor highly related to the serrated pathway, are more common in females and in proximal colon lesions. ${ }^{29}$ Meanwhile, some reports highlight a higher SDR in males, ${ }^{21}$ and in a review with 60,000 colonoscopies, there was no important difference on the prevalence of SLs among men and women. ${ }^{24}$

The SLs were located proximal to the splenic flexure in $68.2 \%$ of the cases; regarding SSLs, the number corresponded to $85.4 \%$ of the lesions. The SDR and SSLDR were also higher when calculated considering only proximal lesions (22.3 and $15.3 \%$, respectively) $(p<0.001)$. This finding may also be associated with the nonresection of diminutive hyperplastic polyps on the distal colon, affecting $10 \%$ of the patients, which reduces the frequency of lesions being removed on the left side. It is known that SLs are more often found on the proximal colon, and that the SSLDR is higher when only proximal lesions are considered. ${ }^{18}$

Among the risk factors associated with SLs, only smoking was linked to a bigger chance of having these lesions (PR: 1.18; 95\%CI: 1.00-1.40). As other studies have already reported, smoking is a risk factor for all types of polyps, but specially for SLs, enhancing twofold the risk of developing them. However, in disagreement with other research, obesity, alcohol intake, and skin color were not associated with a higher risk in our results. ${ }^{30}$
Considering the high detection rates obtained, it is possible to imply that a lot of SLs are being undetected during exams, contributing to the rise in incidence of interval cancer. With a higher SDR and SSLDR, colonoscopy can be as effective in preventing cancer developing from SL on the proximal colon as it already is for AP on the distal colon. $^{18}$

The present study has some limitations: the fact that the colonoscopies were performed by a single experimented endoscopist allows for a more standardized protocol for the performance of exams, helping to have data with less biases; however, the analysis of data embracing results from other specialists, in different hospitals, with different levels of expertise, would bring richer information to be analyzed. Besides the several advantages of a prospective study, the endoscopist was aware of the research, which may have impacted, even if involuntarily, the execution of the exams. Having a single expert pathologist analyzing the samples gives a high rate of identified SLs, but at the same time, considering multiples disagreement between pathologists on the diagnosis of these lesions can also be a limiting factor to our results.

\section{Conclusion}

With the present study, we can conclude that smoking is a risk factor for developing SLs. The SDR and SSLDR are higher than previously suggested, and are even higher when only proximal colon lesions on patients undergoing screening colonoscopy are analyzed. This reassures the need of wellstablished benchmarks for SL detection, so the number of undetected lesions on colonoscopy can decrease.

\section{Conflict of Interests}

The authors have no conflict of interests to declare.

\section{Acknowledgment}

The authors would like to thank Dr. Lix de Oliveira Reis and Dr. Silvio Ciquini for all the support and for proving access to all the data needed fot the research.

\section{References}

1 GLOBOCAN. Cancer Today, Global Cancer Observatory, 2018. Available from: http://gco.iarc.fr/

2 Snover DC. Update on the serrated pathway to colorectal carcinoma. Hum Pathol 2011;42(01):1-10

3 Bettington M, Walker N, Clouston A, Brown I, Leggett B, Whitehall V. The serrated pathway to colorectal carcinoma: current concepts and challenges. Histopathology 2013;62(03):367-386

4 Brenner H, Kloor M, Pox CP. Colorectal cancer. Lancet 2014;383 (9927):1490-1502

5 Nagtegaal ID, Odze RD, Klimstra D, et al; WHO Classification of Tumours Editorial Board. The 2019 WHO classification of tumours of the digestive system. Histopathology 2020;76(02):182-188

6 Moussata D, Boschetti G, Chauvenet M, et al. Endoscopic and histologic characteristics of serrated lesions. World J Gastroenterol 2015;21(10):2896-2904

7 East JE, Atkin WS, Bateman AC, et al. British Society of Gastroenterology position statement on serrated polyps in the colon and rectum. Gut 2017;66(07):1181-1196 
8 Kaminski MF, Regula J, Kraszewska E, et al. Quality indicators for colonoscopy and the risk of interval cancer. N Engl J Med 2010;362 (19):1795-1803

9 Lee HH, Lee BI. Image-Enhanced Endoscopy in Lower Gastrointestinal Diseases: Present and Future. Clin Endosc 2018;51(06): 534-540

10 Arain MA, Sawhney M, Sheikh S, et al. CIMP status of interval colon cancers: another piece to the puzzle. Am J Gastroenterol 2010;105(05):1189-1195

11 Mouchli MA, Ouk L, Scheitel MR, et al. Colonoscopy surveillance for high risk polyps does not always prevent colorectal cancer. World J Gastroenterol 2018;24(08):905-916

12 Zorzi M, Senore C, Da Re F, et al; Equipe Working Group. Detection rate and predictive factors of sessile serrated polyps in an organised colorectal cancer screening programme with immunochemical faecal occult blood test: the EQuIPE study (Evaluating Quality Indicators of the Performance of Endoscopy). Gut 2017;66 (07):1233-1240

13 Anderson JC, Butterly LF, Goodrich M, Robinson CM, Weiss JE. Differences in detection rates of adenomas and serrated polyps in screening versus surveillance colonoscopies, based on the new hampshire colonoscopy registry. Clin Gastroenterol Hepatol 2013;11(10):1308-1312

14 van Herwaarden YJ, Pape S, Vink-Börger E, et al. Reasons why the diagnosis of serrated polyposis syndrome is missed. Eur J Gastroenterol Hepatol 2019;31(03):340-344

15 Lam YF, Leung WK. The Importance of Increased Serrated Polyp Detection Rate. Curr Colorectal Cancer Rep 2016;12:81-87

16 Rex DK, Schoenfeld PS, Cohen J, et al. Quality indicators for colonoscopy. Gastrointest Endosc 2015;81(01):31-53

17 Rex DK, Kahi C, O'Brien M, et al. The American Society for Gastrointestinal Endoscopy PIVI (Preservation and Incorporation of Valuable Endoscopic Innovations) on real-time endoscopic assessment of the histology of diminutive colorectal polyps. Gastrointest Endosc 2011;73(03):419-422

18 Clark BT, Parikh ND, Laine L. Yield of repeat forward-view examination of the right side of the colon in screening and surveillance colonoscopy. Gastrointest Endosc 2016;84(01): 126-132

19 Anderson JC, Butterly LF, Weiss JE, Robinson CM. Providing data for serrated polyp detection rate benchmarks: an analysis of the New Hampshire Colonoscopy Registry. Gastrointest Endosc 2017; 85(06):1188-1194
20 Kahi CJ, Hewett DG, Norton DL, Eckert GJ, Rex DK. Prevalence and variable detection of proximal colon serrated polyps during screening colonoscopy. Clin Gastroenterol Hepatol 2011;9(01): 42-46

21 Ross WA, Thirumurthi S, Lynch PM, et al. Detection rates of premalignant polyps during screening colonoscopy: time to revise quality standards? Gastrointest Endosc 2015;81(03): 567-574

22 Payne SR, Church TR, Wandell M, et al. Endoscopic detection of proximal serrated lesions and pathologic identification of sessile serrated adenomas/polyps vary on the basis of center. Clin Gastroenterol Hepatol 2014;12(07):1119-1126

23 Abdeljawad K, Vemulapalli KC, Kahi CJ, Cummings OW, Snover DC, Rex DK. Sessile serrated polyp prevalence determined by a colonoscopist with a high lesion detection rate and an experienced pathologist. Gastrointest Endosc 2015;81(03):517-524

24 Meester R, Lansdop-Vogelaar I, Ladabaum U. What is the Prevalence of Serrated Colorectal Polyps, and What Does This Imply About Their Cancer Risk: A Systematic Literature Review. Gastroenterology 2019; 156(06):1084-1085. Available from: https://www.sciencedirect.com/ science/article/abs/pii/S0016508519396726

25 Vleugels JL, IJspeert JE, Dekker E. Serrated lesions of the colon and rectum: the role of advanced endoscopic imaging. Best Pract Res Clin Gastroenterol 2015;29(04):675-686

26 Bettington M, Walker N, Rahman T, et al. High prevalence of sessile serrated adenomas in contemporary outpatient colonoscopy practice. Intern Med J 2017;47(03):318-323

27 Schramm C, Mbaya N, Franklin J, et al. Patient- and procedurerelated factors affecting proximal and distal detection rates for polyps and adenomas: results from 1603 screening colonoscopies. Int J Colorectal Dis 2015;30(12):1715-1722

28 Occhipinti P, Saettone S, Cristina S, Ridola L, Hassan C. Correlation between adenoma and serrated lesion detection rates in an unselected outpatient population. Dig Liver Dis 2015;47(06): 508-511

29 Weisenberger DJ, Levine AJ, Long TI, et al; Colon Cancer Family Registry. Association of the colorectal CpG island methylator phenotype with molecular features, risk factors, and family history. Cancer Epidemiol Biomarkers Prev 2015;24(03):512-519

30 Burnett-Hartman AN, Passarelli MN, Adams SV, et al. Differences in epidemiologic risk factors for colorectal adenomas and serrated polyps by lesion severity and anatomical site. Am J Epidemiol 2013;177(07):625-637 